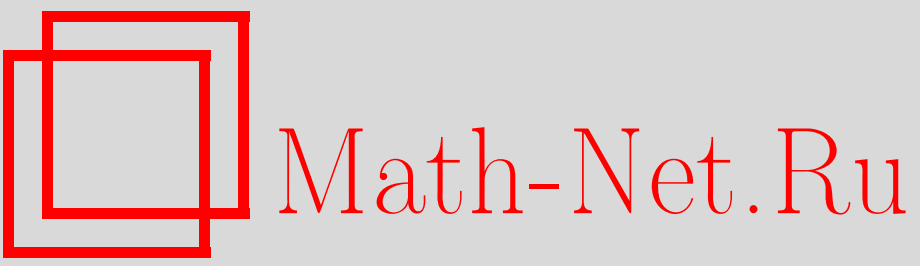

Е. И. Ганжа, С. П. Царев, Алгебраическая формула суперпозиции и полнота преобразований Бэклунда $(2+1)$-мерных интегрируемых систем, УМH, 1996, том 51, выпуск 6, 197-198

DOI: https://doi.org/10.4213/rm1025

Использование Общероссийского математического портала Math-Net.Ru подразумевает, что вы прочитали и согласны с пользовательским соглашением

http: //www. mathnet.ru/rus/agreement

Параметры загрузки:

IP: 3.85 .7 .115

26 апреля 2023 г., $17: 26: 33$ 


\title{
АЛГЕБРАИЧЕСКАЯ ФОРМУЛА СУПЕРПОЗИЦИИ И ПОЛНОТА ПРЕОБРАЗОВАНИЙ БЭКЛУНДА (2+1)-МЕРНЫХ ИНТЕГРИРУЕМЫХ СИСТЕМ
}

\author{
Е. И. ГАНЖА, С.П. ЦАРЕВ
}

Как известно, преобразование Бэклунда для многих $(1+1)$-мерных интегрируемых уравнений обладает свойством нелинейной суперпозиции, позволяюшим по двум решениям $u^{(1)}(x, t)$, $u^{(2)}(x, t)$, полученным из некоторого начального $u^{(0)}(x, t)$, найти с помошью явной алгебраической формулы четвертое решение этого же уравнения $u^{(12)}(x, t)$, связанное преобразованиями Бэклунда с промежуточными $u^{(i)}$. Многие $(2+1)$-мерные интегрируемые системы также обладают (авто)преобразованиями Бэклунда и принципом нелинейной суперпозищии. Однако, соответствующее $u^{(12)}(x, y, t)$, как правило, требует для своего нахождения квадратуры, т.е. алгебраической формулы нет. Мы покажем, как в этом случае получить алгебраическую формулу нелинейной суперпозиции.

Изложение метода мы проведем на примере преобразования Рибокура триортогональных систем координат в $\mathbb{R}^{3}$ - одном из примеров $(2+1)$-мерного геометрического преобразования Бэклунда, а также преобразования Мутара, возникшем впервые в классической дифференциальной геометрии конца XIX века [3], [4] и имеющем многочисленные приложения в теории интегрируемых $(2+1)$-мерных систем [2], [5]. Основой предлагаемого метода является следующая диаграмма преобразований Бэклунда-Рибокура (рис. 1). Здесь $\vec{x}^{(0)}$ - задающая триортогональную систему координат вектор-функция $\vec{x}^{(0)}\left(u^{1}, u^{2}, u^{3}\right)$ - "начальное" решение $(2+1)$-мерной системы Ламе (см. [3], [4]), $\vec{x}^{(i)}$ - его преобразования Рибокура, $\vec{x}^{(i j)}$ получены квадратурами по принципу нелинейной суперпозиции, $\varphi^{(*)}$ потенциалы преобразований Рибокура.

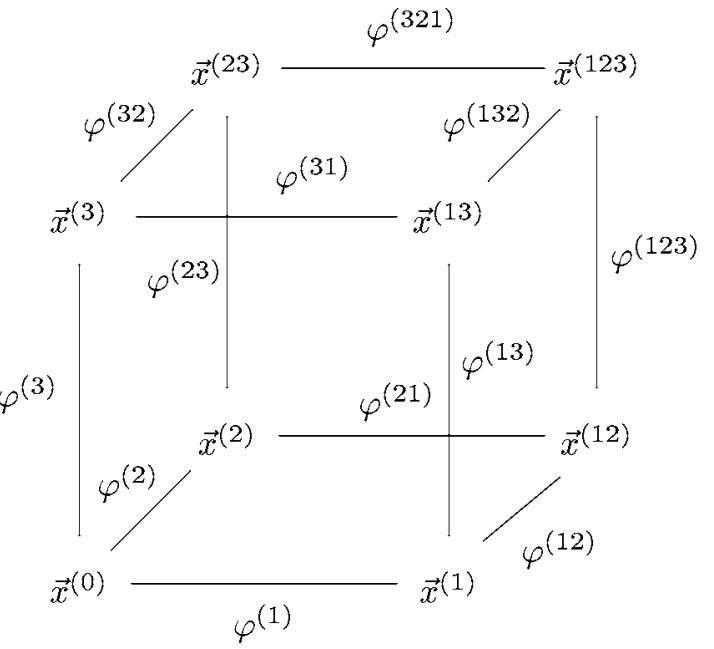

Рис. 1

Как показано в [1], указанная на рис. 1 диаграмма действительно коммутативна для $(1+1)$-мерных уравнений (sine-Gordon, КдФ, система трехволнового взаимодействия). Для $(2+1)$-мерных систем именно диаграмма рис. 1 дает возможность избавиться от квадратур и получить однозначную алгебраическую формулу суперпозиции (“формулу куба”).

Теорема 1. Пусть заданы три преобразования Рибокура $\vec{x}^{(0)} \stackrel{\varphi^{(i)}}{\longrightarrow} \vec{x}^{(i)}, i=1,2,3, u$ полученные из них суперпозициями $\vec{x}^{(12)}, \vec{x}^{(13)}, \vec{x}^{(23)}$. Тогда существует единственная триортогональная система координат $\vec{x}^{(123)}$, связанная преобразованиями Рибокура с $\vec{x}^{(i j)}$ и выражсающаяся через заданные $\vec{x}^{(i)}, \vec{x}^{(i j)}$ алгебраическими формулами.

Для уравнения Мутара $u_{x y}=M(x, y) u(u=u(x, y))$ и его эллиптических и гиперболических вариантов также возможно построить алгебраическую формулу суперпозиции преобразований Мутара на основе диаграммы рис. 1. Фактически Л. Бьянки в [3, v. II, part II, § 297] в несколько другой форме приводит получающуюся "формулу куба".

Как можно проверить, если имеется четыре начальных преобразования Рибокура, мы получаем коммутативную диаграмму преобразований Рибокура (четырехмерный скелет куба),

Работа выполнена при частичной финансовой поддержке Российского фонда фундаментальных исследований (грант № 96-01-00166). 
включающую 16 вершин. Разумно предположить коммутативность аналогичных диаграмм в случае $n$ начальных преобразований Рибокура. Важное свойство таких "гиперкубов Бьянки" состоит в возможности получить широкие классы решений $(2+1)$-мерных интегрируемых систем, используя лишь алгебраические операции (выполнив квадратуры лишь "на первом уровне"). Полученные формулы аналогичны известным “формулам вронскианов и пфаффианов" [2].

Преобразования Бэклунда позволяют построить цепочку решений $\left(u_{0}\right) \rightarrow\left(u_{1}\right) \rightarrow\left(u_{2}\right) \rightarrow$ $\cdots \rightarrow\left(u_{k}\right) \rightarrow \cdots$, выполняя лишь алгебраические операции, начиная с $u_{3}$. Однако вопрос о том, насколько широк получаюшийся набор решений $u_{k}$ (по сравнению с множеством всех решений соответствующих уравнений) оставался открытым. В настоящей работе мы положительно решаем вопрос о (локальной) плотности для случаев уравнений Мутара и Ламе. Тем самым найденные в [2] семейства решений $(2+1)$-мерных интегрируемых уравнений образуют "мошный" запас их решений, а классическое преобразование Рибокура позволяет получить "почти всякую" ортогональную криволинейную систему координат в $\mathbb{R}^{3}$, что позволяет считать системы уравнений, описываюшие эти системы координат (в частности, уравнения $(1),(2))$ вполне интегрируемыми $(2+1)$-мерными системами.

Для преобразований Рибокура воспользуемся следующей формой системы уравнений Ламe [4]:

$$
\left\{\begin{array}{l}
\frac{\partial \beta}{\partial x_{0}}=\lambda^{2} \frac{\partial \alpha}{\partial x_{0}} \\
\frac{\partial \beta}{\partial x_{1}}=-\lambda^{2} \frac{\partial \alpha}{\partial x_{1}} \\
\frac{\partial(\alpha+\beta)}{\partial x_{2}}=(\beta-\alpha) \frac{\partial \log \lambda}{\partial x_{2}}
\end{array}\right.
$$

Исключая $\alpha, \beta$, легко свести (1) к одному уравнению на “потенциал" $M\left(x_{0}, x_{1}, x_{2}\right)=\ln \lambda$ :

$$
\frac{\partial^{3} M}{\partial x_{0} \partial x_{1} \partial x_{2}}=\operatorname{cth}(M) \frac{\partial M}{\partial x_{0}} \frac{\partial^{2} M}{\partial x_{1} \partial x_{2}}+\operatorname{th}(M) \frac{\partial M}{\partial x_{1}} \frac{\partial^{2} M}{\partial x_{0} \partial x_{2}} .
$$

Преобразование Рибокура (2) задается парой решений $\{\alpha, \beta\}$ и $\{\bar{\alpha}, \bar{\beta}\}$ системы (1).

ТЕОрема 2. Пусть дан начальный потенциал $M_{0}\left(x_{0}, x_{1}, x_{2}\right)$-решение $(2)$ в окрестности точки $(0,0,0)$. Тогда для любого $N=0,1,2, \ldots$ найдется такое $K$, что для любого другого потенциала $\bar{M}\left(x_{0}, x_{1}, x_{2}\right)$ найдется цепочка преобразований Рибокура $M_{0} \rightarrow M_{1} \rightarrow M_{2} \rightarrow \cdots \rightarrow M_{K}$ длиньц $K, \partial_{0}^{i} \partial_{1}^{j} \partial_{2}^{k} M_{K}(0,0,0)=\partial_{0}^{i} \partial_{1}^{j} \partial_{2}^{k} \bar{M}(0,0,0)$.

Потенциалы $M(x, y)$ уравнения Мутара - произвольные функции, не удовлетворяюшие какому-либо дифференциальному уравнению.

ТеОРема 3. Пусть задан произвольный начальный потенциал $M_{0}(x, y)$. Тогда для любого $N=0,1,2, \ldots$ найдется такое $K$, что для любого набора чисел $P_{i j}, 0 \leqslant i, j$, $i+j \leqslant N$, производнье потенциала $M_{K}$ (из последовательности преобразований Мутара) в точке $(0,0)$ совпадают с $P_{i j}: P_{i j}=\partial_{x}^{i} \partial_{y}^{j} M_{K}(0,0)$.

Доказательства приведенных результатов доступны через электронный архив solv-int@xyz.lanl.gov: solv-int/9606001, solv-int/9606002, solv-int/9606003.

\section{СПИСОК ЛИТЕРАТУРЫ}

[1] Царев С.П. Дифференциально-геометрические методы интегрирования систем гидродинамического типа // Дис. ... докт. физ.-матем. наук. М., 1993. [2] Athorne C., Nimmo J. J. C. // Inverse Problems. 1991. V. 7. P. 809-826. [3] Bianchi L. Lezioni di geometria differenziale. V. I, II. Bologna: N. Zanichelli, 1923-1927. [4] Darboux G. Leçons sur les systèmes orthogonaux et les coordonnées curvilignes. Paris: Gauthier-Villars, 1910. [5] Oevel W., Rogers C. // Rev. Mod. Phys. 1993. V. 5. P. 299-330.

Московский государственный университет им. М. В. Ломоносова; Красноярский государственный педагогический университет
Принято редколлегией 17.07 .1996 